\title{
Review of: "The effects of rapid mass vaccination against SARS-CoV-2 and its Variants-of-Concern: Evidence from an early VoCs hotspot"
}

Nir Ben-Tal ${ }^{1}$

1 Tel Aviv University

Potential competing interests: The author(s) declared that no potential competing interests exist.

This is a carefully conducted statistical survey of the effect of the biontech/pfizer anti-covid vaccine. It is based on mass vaccination operation that took place in Schwaz District, Austria, where most of the over 16 years old population (totaling 100,000 people) was given the first dose of the vaccine between March 1621 . With that, a total of $70 \%$ of the population got vaccinated, compared to $10 \%$ before that. The situation before the vaccination, as well as the situation in nearby Districts, also with population that was also $10 \%$ vaccinated, were used as references. The results convincingly show the positive effect of the vaccine on number of infected individuals, number of hospitalized individuals, and number of intensive care unit cases. Most importantly, the study clearly shows that the vaccine is also effective against the two harmful covid variants, B.1.351 and B.1.1.7/E484K.

The study was conducted very professionally, with many controls, and the findings are presented clearly and objectively. The Discussion outlines various possibilities for confounding factors that might bias the results. I am convinced.

A minor suggestion: the term "VoC" should be defined on first use. Ideally already in the title, but if not, at least in the Abstract. 